\title{
Effectiveness of Intensive Perioperative Nutrition Therapy Among Adults Undergoing Gastrointestinal And Oncological Surgery In Public Hospital: Study Protocol For A Pragmatic Randomised Control Trial.
}

\section{A'ishah Zafirah Abdul A'zim}

Department of Dietetics, Faculty of Medicine and Health Sciences, Universiti Putra Malaysia, Selangor, Malaysia. Department of Dietetics, Hospital Pengajar Universiti Putra Malaysia, Selangor, Malaysia Zalina Abu Zaid ( $\nabla$ zalina@upm.edu.my)

Universiti Putra Malaysia Fakulti Perubatan dan Sains Kesihatan https://orcid.org/0000-0002-95725148

\section{Barakatun Nisak Mohd Yusof}

Department of Dietetics, Faculty of Medicine and Health Sciences, Universiti Putra Malaysia, Selangor, Malaysia. Department of Dietetics, Hospital Pengajar Universiti Putra Malaysia, Selangor, Malaysia

\section{Mohd Faisal Jabar}

Department of Surgery, Faculty of Medicine and Health Sciences, Universiti Putra Malaysia, Selangor, Malaysia

\section{Aainaa Syarfa Mohd Shahar}

Department of Dietetics, Faculty of Medicine and Health Sciences, Universiti Putra Malaysia, Selangor, Malaysia. Department of Dietetics, Hospital Pengajar Universiti Putra Malaysia, Selangor, Malaysia

\section{Study protocol}

Keywords: perioperative nutrition therapy, early nutrition therapy, surgical patients, post-operative outcomes, oral nutrition support

Posted Date: September 14th, 2021

DOI: https://doi.org/10.21203/rs.3.rs-795156/v1

License: (9) This work is licensed under a Creative Commons Attribution 4.0 International License. Read Full License 


\section{Abstract}

Background: Perioperative malnutrition is common in patients undergoing gastrointestinal-oncology surgical and associated with longer hospital stays, increased post-operative complications, poorer quality of life and lower survival rate. Current practice emphasises the roles of early perioperative nutrition therapy as early intervention in order to combat the post-operative complications of patients and the implementation is now widely adopted. The aim of the study is to determine the effects of intensive perioperative nutrition therapy to overcome the post-operative complications in adult patients undergoing gastrointestinal and oncological surgery.

Methods: This is a pragmatic randomised control trial study where elective admitted patients will be randomised into Intervention (SS) or Control (NN) Group. All data will be collected during a face to face interview, anthropometric measurement, blood sampling (albumin, white blood count, haemoglobin and creactive protein), handgrip strength and post-operative complications. Group SS will be receiving a tailored lifestyle and intensively supplemented with oral nutrition support as compared with Group NN that receive standard medical care. The primary outcomes are length of stay in hospital, bowel function and solid food toleration. Additional outcome measures are changes in biochemical profile, nutritional and functional status. The effects of intervention between groups towards outcome parameters will be analyse by using the SPSS General Linear Model (GLM) for repeated measure procedure.

Discussion: The intervention implemented in this study will serve as a baseline data in providing an appropriate nutritional management in patients undergoing gastrointestinal and oncological surgery.

Trial registration: ClinicalTrials.gov Protocol Registration and Results System (PRS), NCT04347772. Registered 20 November 2019, https://www.clinicaltrials.gov

\section{Background}

Malnutrition is well reported in surgery patients $(1,2)$ especially those who undergone major surgery $(6,7$, 8 ) and particularly at risk in patients undergoing surgery for upper gastrointestinal or colorectal cancer (4, 5). Pre-operative malnutrition shows increase length of hospital stay, higher rate of surgical site infection and mortality (9) and associated with higher post-operative complication, increased costs, poorer quality of life and lower survival rate $(9,10)$. Studies show high prevalence of malnutrition or high nutritional risk during hospital admission $(11,12)$ but this is rarely assessed in the clinical setting especially patients who will undergone elective surgery.

Nutrition requirement for surgery is higher if compared with normal requirement in order to support speedy recovery. Suboptimal nutritional intake will cause further depletion of patient's nutritional status pre-operatively and higher risk of postoperative complications. Interestingly, one of Malaysian study shows significant number of post-surgery complications compared to pre-surgery which was associated to the poor level of nutrition (10). 
Nutrition therapy is the provision of nutrition or nutrients either orally (regular diet, therapeutic diet, e.g. fortified food, oral nutritional supplements) or via enteral nutrition (EN) or parenteral nutrition (PN) to prevent or treat malnutrition (13) which include dietary counseling. Nutritional therapy is important as it affects mainly the perioperative maintenance of nutritional state in order to prevent postoperative complications (14). It is strongly recommended not to wait until severe disease-related malnutrition has developed, but to start nutrition therapy early, as soon as a nutritional risk becomes apparent (3).

In addition, patients with severe nutritional risk in surgical patients with oral feeding, some improvements were shown in shortened duration of hospital stay and flatus, and some of wound and infectious complications (15). Current practice emphasises the roles of early nutrition therapy as early intervention in order to combat the post-operative complications of patients and the implementation is now widely adopted. However, there is a very limited data, locally to present the effects of perioperative nutrition therapy as well as process of nutrition management, from screening/assessment on admission to nutritional support and monitoring for surgical patients. Therefore, there is a need to study in this area in order to find good practices and should be mandated in routine patient care regardless of disease type.

\section{Methods/design}

\section{Design}

This is a pragmatic randomised control trial (RCT), that will be conducted among sixty-eight adult patients undergoing major elective surgery (gastrointestinal and oncological surgery) in Hospital Serdang, Malaysia. The RCT conformed to the Consolidated Standards of Reporting Trials (CONSORT) statement for reporting RCT with two arms comparing an intervention group with a control group (17). After consenting to participate, subjects were allocated into intervention (SS) and control (NN) groups randomly. The flow diagram for recruitment and randomisation is shown in Fig. 1. A graphical presentation of the proposed study procedure and timeline is shown in Fig. 2. The schedule of enrolment, interventions and assessments in Table 1 (18).

TABLE 1 The schedule of enrolment, interventions and assessments 


\begin{tabular}{|c|c|c|c|c|c|c|}
\hline & \multicolumn{6}{|c|}{ STUDY PERIOD } \\
\hline & Enrolment & Allocation & & Allo & & Close-out \\
\hline TIMEPOINT ** & $-t_{1}$ & $\mathbf{0}$ & $t_{1}$ & $t_{2}$ & $t_{3}$ & $t_{x}$ \\
\hline ENROLMENT: & & & & & & \\
\hline Eligibility screen & $\mathrm{X}$ & & & & & \\
\hline Informed consent & $\mathrm{X}$ & & & & & \\
\hline Allocation & & $\mathrm{X}$ & & & & \\
\hline INTERVENTIONS: & & & & & & \\
\hline [Intervention group] & & & & & & \\
\hline [Control group] & & & & & & \\
\hline ASSESSMENTS: & & & & & & \\
\hline $\begin{array}{r}\text { [sociodemogrphic, } \\
P G-S G A, \text { nutritional } \\
\text { and functional status] }\end{array}$ & $\mathrm{X}$ & $\mathrm{X}$ & & & & \\
\hline $\begin{array}{l}\text { [complications of } \\
\text { post-operative] }\end{array}$ & & & & $\mathrm{X}$ & & $\mathrm{X}$ \\
\hline
\end{tabular}

\section{Study population}

Men and women who planned for surgical procedures will be invited to participate in this study with informed consent. A screening form will be used to exclude those who do not meet the inclusion criteria. The inclusion criteria are those who receiving elective major surgery treatments for gastrointestinal and oncology-related surgery, aged from 18 years old to 65 years old, Malaysian, able to communicate verbally, Malnutrition Screening Tool (MST) score $\geq 2$ and provided and signed informed consent. The exclusion criteria are those who are receiving elective major surgery treatments other than gastrointestinal and oncology-related including bariatric surgery, those who had received pre-operative enteral or PN, those who requiring emergency surgery, complicated with chronic diseases and fluid retention (renal/ cardiovascular/pulmonary/ hepatic) and those who participated in other research study. 


\section{Recruitment}

Potential patients who are attend surgical clinic will be approached by the project team member. They will inform the study procedures to all potential participants. However, the study is single-blinded is when the investigator but not the study participants know which treatment has been allocated. The patient information sheet and consent form will be given to eligible subjects who agreed to be recruited in the study. Subjects will be allowed to bring back consent form if she/he keen to seek advice from family member. The consented participants will be randomised to one of two groups by means of sealed envelope into SS or NN.

\section{Randomisation}

Permuted block randomisation with Body Mass Index (BMI) and gender stratification will be conducted to assign participants to either the intervention or control group. Randomisation will be based on block method for block size of four, with A for intervention group and B for the control group. This block of four had six different possible arrangements of two As and Bs. It will be performed using software application i.e.https://www.sealedenvelope.com/simple-randomiser/v1/lists (19). The flow diagram for recruitment and randomisation is shown in Fig. 1.

To guarantee allocation concealment, the randomisation will be carried out by an independent third party. The envelope will be opened on the spot, deciding which group the participant would be allocated to either Group SS: Intervention Group or Group NN: Control Group. The Group SS will be matched to Group $\mathrm{NN}$ for BMI and gender.

\section{Study procedure}

All data will be collected during a face to face interview, blood sampling and direct anthropometric measurement with the participants at Hospital Serdang starting from before operation until two weeks after discharge.

\section{Intervention group, Group SS}

Group SS will be received a tailored, more intensive and ongoing nutrition support and lifestyle advice as compared with Group NN. They will be supplemented with oral nutrition support (ONS), available in $400 \mathrm{~g} / \mathrm{can}$, providing $226 \mathrm{kcal} /$ serving and $9.6 \mathrm{~g}$ protein/serving. ONS will be given according to patient's requirement, with maximum of 4 serving/day. Patients are encouraged to consume ONS in small, frequent and in between meals.

\section{Control group, Group NN}

While participants in Group NN which referred as control group will be received the standard care of the clinic without supplemented with ONS. Nutritional advice will be based on a guideline specifically focused on treatment of symptoms such as nausea, vomiting, loss of appetite and diarrhea, and how to deal with the symptoms through nutritional approaches. Basically, the advice will be given by clinician or 
nurses in the clinic. Those who are identified as having moderate and severe malnutrition will be referred to a dietitian for nutrition counselling.

All participants will be received standard post-operative care from clinical and nurse staff with commencement of free fluids and reintroduction of normal diet without interference by the researcher or protocol. In addition to Group SS, ONS will be given after surgery, starting at the first day of nourishing fluid/diet allowance until the day of hospital discharge according to the prescription given. All participants will have followed up visit either at outpatient clinic or home visit, 4 weeks after surgery.

Participants will be monitored for complications throughout the study period. Monitoring will be taken place by daily observation of the participant, by checking their medical and nursing notes, and by liaison with the attending surgical team and nursing staff. While complications will be noted as major or minor by using validated criteria (20).

\section{Criteria for withdrawal}

The participation will be voluntary, and participants will be free to withdraw from the study at any time without giving any reason and this will in no way affect the participants' future treatment.

\section{Data collection procedure}

Each participant will be assessed in clinic/wards on four different sessions (Baseline, first, second and third visits) over the study period (Fig. 2). During baseline visit, screening and recruitment will be conducted at surgical clinic, attending by potential participants who are having appointment for examination and to get surgery appointment. Potential participants are those who are scheduled to have surgery and based on inclusion and exclusion criteria. Scheduled project team member will be assigned to approach and inform with study procedures to all potential participants. The patient information sheet and consent form will be given to eligible subjects who agreed to be recruited in the study. Subjects are allowed to bring back consent form if she/he keen to seek advice from family member. Agreed and consented participant will be randomly sampled and will be divided into 2 groups.

During baseline visit (re-study phase), the researcher will be obtained patients' history of dietary intake via 24-hour dietary recall. Based on this assessment, the researcher will be able to determine participants' estimated intake based on their estimated requirements. The dietary advice will be given individually and was structured based on ESPEN Guideline for surgical patients (3).

Participants who are allocated to Group SS will be supplied with ONS, maximum of 4 servings/day according to their requirement. Participants need to consume ONS given starting from recruitment until 6 hours before surgery. Follow up through phone call from time to time will be done to assess participants' compliance towards ONS given. While during study phase, participants need to consume ONS once allowed, and compliance will be assessed daily until discharge. Participants will be asked to keep the ONS packaging material during both phases. Researcher will collect the packaging material back during 
1 st visit and the day of hospital discharge. While Group NN will be not supplemented any of ONS in prestudy phase and study phase.

During visit 1 , and visit 3, dietary intake data will be collected using a self-administered 2-days 24-hour dietary record. All the participants will also be given comprehensive verbal instructions on the method of recording food intake using the 2-days 24-hour dietary record. The participants who were less educated will be asked to seek assistance from their family members to record for them. They will be asked to complete the 2-days 24-hour dietary record of food intakes before their next visit, which was used as the basis for their individualised dietary advice.

During baseline visit, all of data collections will be conducted including socio-demographic questionnaire, with exception of data for complications post-operative which only can be obtained at visit 2 (length of hospital stays, length of bowel function and length of solid food toleration). Meanwhile, anthropometric measurements (except height) and PG-SGA questionnaire, the same methods and instruments will be used repeatedly at visit 1 and visit 3 . Data for handgrip will be conducted at every visit while biochemical data will be collected for second time at the end of the study which is during visit 3 . The details of the assessment and procedure are shown in Table 2. 
Table 2

The assessment procedure at (baseline), Visit 1, Visit 2, and Visit 3

\begin{tabular}{|c|c|c|c|c|}
\hline \multirow[t]{2}{*}{ The assessment procedure } & \multicolumn{4}{|l|}{ Visit } \\
\hline & Baseline & 1 & 2 & 3 \\
\hline \multicolumn{5}{|l|}{ Questionnaire } \\
\hline Social demographic background & / & & & \\
\hline The scored PG-SGA & I & & / & / \\
\hline \multicolumn{5}{|l|}{ Dietary assessment } \\
\hline 1-day 24-hour dietary recall & / & & & \\
\hline 2-day 24-hour dietary record & & & / & / \\
\hline \multicolumn{5}{|l|}{ Anthropometric measurements } \\
\hline Body weight & / & & / & / \\
\hline Height & / & & & \\
\hline Mid arm circumference & / & & / & / \\
\hline Triceps skinfold thickness & / & & / & / \\
\hline \multicolumn{5}{|l|}{ Biochemical data } \\
\hline Serum albumin - Medical report & / & & & / \\
\hline White blood count - Medical report & / & & & / \\
\hline Haemoglobin - Medical report & / & & & / \\
\hline C-reactive protein & / & & & / \\
\hline \multicolumn{5}{|l|}{ Functional status } \\
\hline Handgrip strength & / & / & / & / \\
\hline \multicolumn{5}{|l|}{ Complications post-operative } \\
\hline Length of stay in hospital & & & / & \\
\hline Length of bowel function & & / & & \\
\hline Length of solid food toleration & & / & & \\
\hline
\end{tabular}

\section{Primary outcome measures}

Post-operative complication: length of stay (LOS) in hospital, length of bowel function and length of solid food toleration will be recorded post-operatively. 


\section{Other variables}

\section{Nutritional status}

\section{The scored Patient-Generated Subjective Global Assessment (PG-SGAC)}

The scored PG-SGAC consisted of two sections with seven domains. The first section, completed by the participant, was in the form of a checklist and comprised weight, food intake, symptoms, activities, as well as function. The second section will be completed by the researcher as it covered the disease and its relation to nutritional requirements, determination of metabolic demands, and was followed by a nutrition-related physical examination, including subjective body composition (i.e. fat, muscle, and fluid status).

A score ranging from 0 to 4 will be given for each domain depending on the impact on the nutritional status. PG-SGAC scores that ranged from 0 to 35 reflected a greater risk of malnutrition or indicated a lower nutritional status of the patient. These scores will be transformed into global ratings - Stage A, Stage B and Stage C - which represented the states of being well-nourished, moderately malnourished, and severely malnourished, respectively. Then, the criticality of need for nutritional intervention will be identified and classified based on the scores with 0 to 1 point requiring no intervention, health education for 2 to 3 points, dietetic intervention for 4 to 8 points, and nutrition support for $\geq 9$ points.

\section{Anthropometric measurements}

The anthropometric measurements will be carried out in this study included body weight, height, mid arm circumference (MAC) and triceps skinfold thickness (TSF). These measurements will be taken directly before the interview with all the participants.

Body weight and height measurements will be taken with participants being shoeless and wearing lightweight clothing with empty pockets, without watches, or other accessories. Weight will be determined to the nearest $0.1 \mathrm{~kg}$ using a digital weighing scale (SECA, British Indicators Ltd., United Kingdom). The machine will be calibrated every morning with a standard weight before it will be used. Height will be measured in the standing position to the nearest $0.1 \mathrm{~cm}$ using a SECA 206 microtoise tape (Vogel and Halke GmbH \& Co, Hamburg, Germany) which will be attached to the wall. The participants will be asked to stand straight with the head in the Frankfort plane, feet together, knees straight, and heels, buttocks, and shoulder blades in contact with the vertical surface of the wall.

MAC and TSF will be taken without any sleeve at measured arm, watches or other accessories. Subject is in a relaxed standing position with the arms hanging by the sides. MAC will be measured in the middle arm (same distant) from acromiole and radiale bone, to the nearest $0.1 \mathrm{~cm}$ using SECA 201 circumference measuring tape (SECA, British Indicators Ltd., United Kingdom). While, TSF will be measured at the most posterior part of the triceps when viewed from the side at MAC level, to the nearest 
0.1 mm using Harpenden skinfold caliper (HaB International Ltd, United Kingdom). The caliper will be calibrated every morning before it will be used to minimize error during measurement. All anthropometric measurements will be taken twice by the same investigators, and the average will be used. Weight and height of the participants will be then used to calculate the BMI (21).

\section{Biochemical data}

Data on serum albumin, white blood count (WBC) and haemoglobin level will be obtained from patients' medical report at baseline and at the end of visit. For other blood parameters, $10 \mathrm{ml}$ of venous blood will be taken following overnight fast. The samples will be separated immediately by means of centrifugation ( $1800 \mathrm{~g}$ for 10 minutes at $4^{\circ} \mathrm{C}$ ). The serum will be stored at $80^{\circ} \mathrm{C}$ until analysis for the measurement of Creactive protein, which will be performed after all samples are collected.

\section{Dietary intake}

Dietary intake will be measured through a 24-hour dietary recall at baseline and two days of 24-hour dietary records during visit 2 and 3 . The participants will be required to keep record of all food and beverage intakes within each 24-hour period of data collection. The participant will be asked their intake before visit. Details of food information and descriptions, which included brand names, preparation and cooking methods, as well as recipes of any mixed dishes eaten during the study period, will be also recorded. In situations where foods and beverages will be consumed away from home, the participants will be encouraged to describe the quantities of foods and beverages consumed using the household measures as well (e.g: glass, cup, Chinese rice bowl, plate, tablespoon, and teaspoon).

Both groups will be provided an explanation by the researcher on how to record their two-day intake in a food diary. They will also be given a detailed set of instructions together with a food album. The food album lists commonly consumed food and includes details of portion sizes relative to typical household measurements to facilitate recalls of serving size and improve accuracy.

A computerised local dietary analysis program, Nutritionist Pro version 2.0 (First Data Bank, The Hearst Corp. United States of America [USA]) will be used to analyse the nutrient intakes of the patients. The foods and beverages consumed by the participants will be coded by type and amount and analysed for nutrient content primarily based on the Malaysian Food Composition Database (MYFCD) (22). Nutritionist Pro will be then used to calculate dietary intake at baseline (based on one 24-hour dietary recall), and at other subsequent assessment time points (based on average intake over each 2-day period of dietary record).

\section{Handgrip strength}

Handgrip strength is measured on the non-dominant hand using Jamar hand dynamometer (Fred Sammons Inc, Burr Ridge, Illinois, USA). Handgrip strength as a surrogate marker for muscle strength in patients with cancer has been well documented elsewhere. Subjects will be sat with their shoulder adducted and neutrally rotated, elbow flexed at $90^{\circ}$, forearm in neutral position, and standard verbal instructions will be given to the subjects to squeeze the dynamometer as hard as possible for three times 
after an interval of 5 seconds in between grips. Average of three successive attempts will be used as the final result.

\section{Secondary outcome measures \\ Participant details and demographics}

Information will be obtained on address, contact number, date of birth, age, sex, ethnic group, education level, job status, income level and marital status.

\section{Medical history}

Investigation of participant's medical history on the type of disease, duration, treatment obtained, and medication received.

\section{Statistical analysis}

\section{Sample size calculation}

This sample size was calculated based on data from a study conducted in colorectal cancer patients undergoing surgery (23). The primary outcome was calculated on length of hospitalised stay between two groups (3.45 days). Assuming a clinically significant difference of 5 units or more in one group relative to the other with a power $(\beta)$ of $90 \%$ and a probability (a) of 0.05, 26 participants have to be included in each group. Allowing for a 'drop-out' rate of $30 \%$, we therefore aim to recruit 34 participants will be included in each group with the final sample size calculated is 68 participants.

\section{Analysis of results}

The data collected will be analyzed by using the statistical software Statistical Package for Social Sciences (SPSS) and checked for normality via Kolmogorov-Smirnov analysis. All data will be normally distributed as indicated by $p>0.05$ unless otherwise stated. If the data will be not normally distributed, analyzes will be carried out on the natural logarithm of the values to improve the symmetry and homoscedasticity of the distribution.

Descriptive statistics, including percentages, mean values, and standard deviations, will be used to describe the baseline demographic data, type of diseases, nutritional status level including anthropometric data, and dietary intake. The values from both groups were compared by using an ANOVA test for approximately normally distributed data, followed by tests on pairs of groups using the Bonferroni adjustment. Non-normal data will be compared by the Kruskal-Wallis test followed by MannWhitney $U$ test. ANCOVA or logistic regression will be used with models adjusted according to potential confounders in order to determine predictors of changes in the key outcome variables of length of hospital stay with $p<0.05$ significance level.

\section{Discussion}


Globally, hospital malnutrition is under-recognized, under-diagnosed and under-treated by health care professional, which may potentially of omission of nutritional assessments in routine patient assessment on admission (24). The standardised process of nutrition assessment will be measured for both the intervention and control groups in the study which will be intention to be used in routine patient care.

The provision of early nutrition intervention or perioperative enteral nutrition in the surgical patient is to optimise patient's nutritional status, prevention and treatment of catabolism, lower the incidence of minor complications during surgical and cost effective (25). In addition, appropriate perioperative nutrition therapy has been shown on improvement of perioperative outcomes especially in gastrointestinal and oncologic surgical patients (2).

Moreover, most studies have investigated the effect of nutrition therapy on post-operative complication in which ONS were prescribed during the pre-operative but not after the surgery even though post-operative ONS also has been shown to have beneficial effect on outcome. The intensive perioperative nutrition therapy has a focus on this outcome which is aimed to overcome the pre and post complications because its efficacy in improving the nutritional status of the participants has been observed.

In parallel, the use of ONS, together with voluntary food intake, as a means of providing nutritional support to surgical patients is more straightforward, easy to administer, comparatively cheap, free from complication and, with the range of flavors now available, palatable $(25,26)$. The intensive perioperative nutrition therapy will be a good choice to be used among the dietitians in clinical setting in order to provide a good outcome of the surgical patients.

\section{Trial Status}

The protocol version number NCT04347772 and date of registration: 20 November 2019. The date of recruitment on 31 August 2021 and the date of recruitment completed on 31 December 2021.

\section{Abbreviations}

EN Enteral nutrition

PN Parenteral nutrition

RCT Randomized Control Trial

CONSORT Consolidated Standards of Reporting Trials

SS Intervention Group

NN Control Group

MST Malnutrition Screening Tool 
BMI Body Mass Index

ONS oral nutrition support

ESPEN The European Society for Clinical Nutrition and Metabolism

LOS length of stay

PG-SGA@ Patient-Generated Subjective Global Assessment

MAC mid arm circumference

TSF triceps skinfold thickness

WBC white blood count

$\beta$ power

a probability

SPSS Statistical Package for Social Sciences

MREC Malaysia Research and Ethics Committee

\section{Declarations}

\section{Ethics approval and consent to participate}

The study protocol was approved by the Malaysia Research and Ethics Committee, Ministry of Health Malaysia (MREC) with ethic approval number NMRR-18-2625-43546 (IIR) and was registered on ClinicalTrials.gov with identity number NCT04347772 and date of registration: 20 November 2019. Written informed consent was obtained from the patients prior to data collection

Consent for publication

Not applicable

Availability of data and materials

The datasets used and/or analysed during the current study are available from the corresponding author on reasonable request.

Competing interests

The authors declare that there is no competing of interest. 
This work was granted from Universiti Putra Malaysia (GP-IPM/2018/9613500)

Authors' contribution

AZAA, ZAZ, BNMY and MFJ conceptualized and design of the study. AZAA \& ASMS conducts the data collection research. ZAZ, BNMY and MFJ provided supervision in the design and execution of the study. AZAA wrote the first of the manuscript. ZAZ, BNMY and MFJ reviewed and edited the manuscript. All authors provided comments, read and approved the final manuscript.

Acknowledgements

The authors wish to express their sincere gratitude to all enthusiastic study participants who extended their cooperation during this study. The authors would like to thank the Director of Health Malaysia for permission to publish this manuscript.

\section{References}

1. Evans DC, Martindale RG, Kiraly LN, Jones CM. Nutrition optimization prior to surgery. Nutr Clin Pract 2014; 29(1):10-21.

2. Williams JD, Wischmeyer PE. Assessment of perioperative nutrition practices andnattitudes-A national survey of colorectal and GI surgical oncology programs. Am J Surg 2017; 213(6):1010-1018.

3. Weimann A, Braga M, Carli F, Higashiguchi T, Hubner M, Klek S, Laviano A, Ljungqvist O, Lobo DN, Martindale R, et al. ESPEN Guideline: Clinical Nutrition in Surgery. Clin Nutr 2017; 36(3):623-650.

4. Farreras N, Artigas V, Cardona D, Rius X, Trias M, González J A. Effect of early postoperative enteral immunonutrition on wound healing in patients undergoing surgery for gastric cancer. Clin Nutr 2005; 24(1):55-65.

5. Nitenberg G, Raynard B. Nutritional support of the cancer patient: issues and dilemmas. Crit. Rev. Oncol. Hematol. 2000; 34(3): 137-168.

6. Young LS, Huong PT, Lam NT, Thu NN, Van HT, Hanh HL, Tuyen le D, Lien DT, Hoc TH, Tuyet CT, et al. Nutritional status and feeding practices in gastrointestinal surgery patients at Bach Mai Hospital, Hanoi, Vietnam. Asia Pac. J. Clin. Nutr. 2016; 25:513-520.

7. Leandro-Merhi V A, de Aquino J L, Sales Chagas J F. Nutritional status and risk factors associated with length of hospital stay for surgical patients. JPEN J Parenter Enteral Nutr 2011; 35: 241-248.

8. Singh H, Watt K, Veitch R, Cantor M, Duerksen DR. Malnutrition is prevalent in hospitalized medical patients: Are house staff identifying the malnourished patient? Nutrition 2006; 22(4):350-354. 
9. Khan $\mathrm{OH}$, Khan $\mathrm{AH}$, Zakaria AD, Hashim MN, Syed Sulaiman SA. Prevalence of malnutrition in surgical population and its impact over early post-operative outcomes at a tertiary care hospital in Malaysia. Malays. J. Pharm. Sci. 2015; 13(1): 71.

10. Nur SMAL, Nurulumi A, Farida I. Complications associated with malnutrition in elective surgical patients in a Malaysian setting. Trop J Pharm Res 2016; 15(6):1321-1325.

11. Barker LA, Gout BS, Crowe TC. Hospital Malnutrition: Prevalence, identification and impact on patients and the healthcare system. Int. J. Environ. Res. Public Health 2011; 8(2):514-527.

12. Garth A K, Newsome CM, Simmance N, Crowe TC. Nutritional status, nutrition practices and postoperative complications in patients with gastrointestinal cancer. J Hum Nutr Diet 2010; 23(4):393-401.

13. Cederholm T, Barazzon R, Austin P, Ballmer P, Biolo G, Bischoff SC, Compher C, Correia I, Higashiguchi T, Holst $\mathrm{M}$, et al. ESPEN guidelines on definitions and terminology of clinical nutrition. Clin Nutr 2017; 36(1):49-64.

14. Chambrier C, Sztark F. French clinical guidelines on perioperative nutrition. Update of the 1994 consensus conference on perioperative artificial nutrition for elective surgery in adults. J Visc Surg 2012; 149(5):e325-336.

15. Choi W J, Kim J. Nutritional care of gastric cancer patients with clinical outcomes and complications: a review. Clin Nutr Res 2016; 5(2):65-78.

16. Horgan A. Pre-operative nutrition support in patients undergoing gastrointestinal surgery. Tech Coloproctol 2014; 18(11):1137-1138.

17. Moher D, Hopewell S, Schulz K F, Montori V, Gøtzsche P C, Devereaux P J, Elbourne D, Egger M, Altman D G. CONSORT 2010 Explanation and elaboration: Updated guidelines for reporting parallel group randomised trials. J. Clin. Epidemiol 2010; 63:e1-e37.

18. Chan A-W, Tetzlaff JM, Gøtzsche PC, Altman DG, Mann H, Berlin J, Dickersin K, Hróbjartsson A, Schulz KF, Parulekar WR, Krleža-Jerić K, Laupacis A, Moher D. SPIRIT 2013 Explanation and Elaboration: Guidance for protocols of clinical trials. BMJ. 2013;346:e7586

19. Sealed Envelope Ltd. 2020. Create a blocked randomisation list. Internet: https://www.sealedenvelope.com/simple-randomiser/v1/lists (accessed 4 August 2020).

20. Buzby GP, Knox LS, Crosby LO, Eisenberg JM, Haakenson CM, McNeal GE, Page CP, Peterson OL, Reinhardt GF, Wiliford WO. Study protocol: a randomized clinical trial of total parenteral nutrition in malnourished surgical patients. Am. J. Clin. Nutr. 1988; 47(2 Suppl):366-381.

21. World Health Organization. Appropriate body-mass index for Asian populations and its implications for policy and intervention strategies. Lancet 2004; 363:157-63. 
23. Dag A, Colak T, Turkmenoglu O, Gundogdu R, Aydin S. A randomized controlled trial evaluating early versus traditional oral feeding after colorectal surgery. Clinics 2011; 66(12): 2001-2005.

24. Woien H, Bjork IT. Nutrition of the critically ill patient and effects of implementing a nutritional support algorithmn in ICU. J. Clin. Nutr. 2006; 15: 168-177.

25. Smedley F, Bowling T, James M, Stokes E, Goodger C, O'Connor O, Oldale C, Jones P, Silk D.

Randomized clinical trial of the effects of preoperative and postoperative oral nutritional supplements on clinical course and cost of care. Br J Surg 2004; 91(8): 983-990. 50

26. MacFie J, Woodcock NP, Palmer MD, Walker A, Townsend S, Mitchell CJ. Oral dietary supplements in pre- and postoperative surgical patients: a prospective and randomized clinical trial. Nutrition 2000; 16(9):723-728.

\section{Figures}




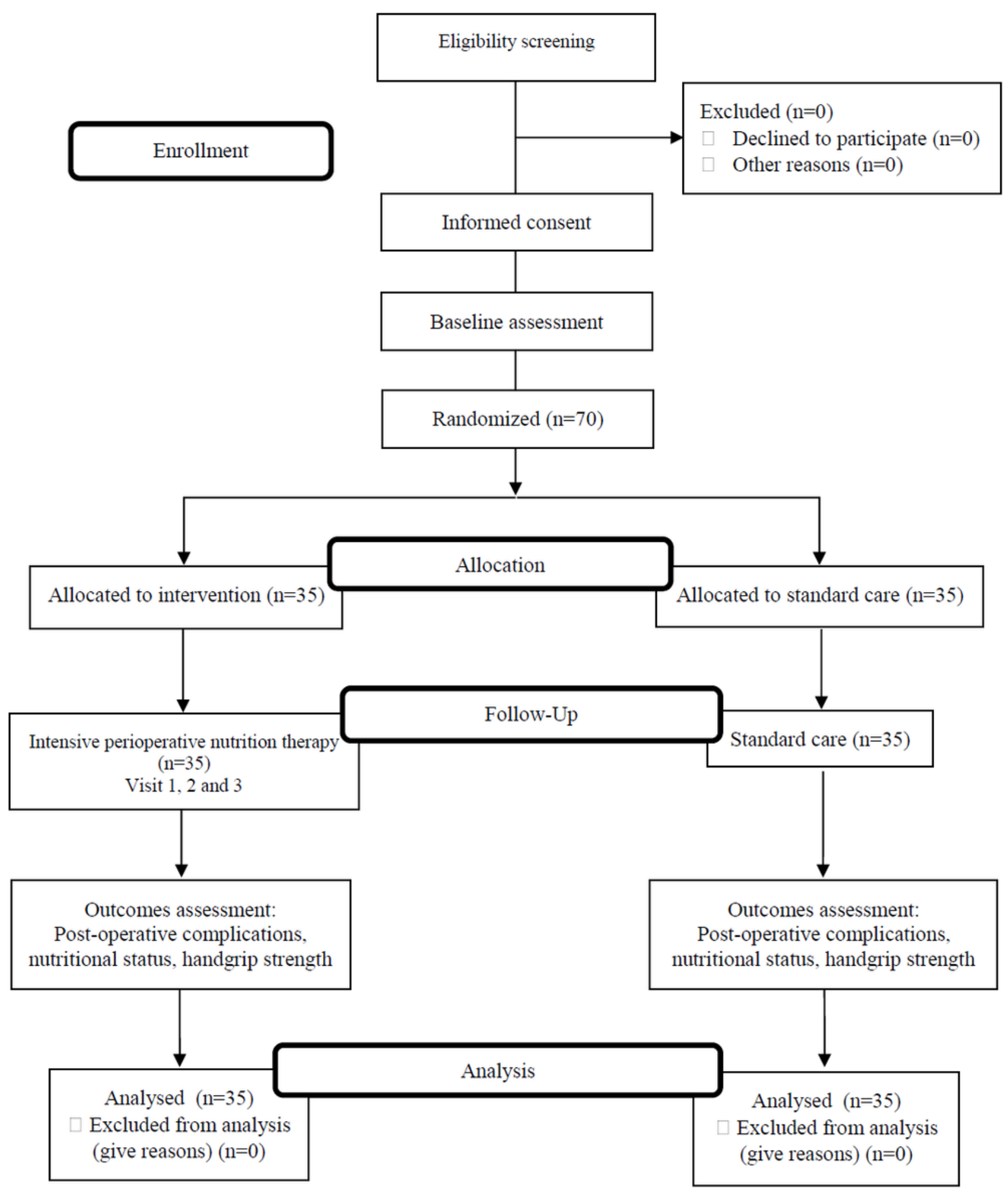

\section{Figure 1}

\section{Flow diagram}




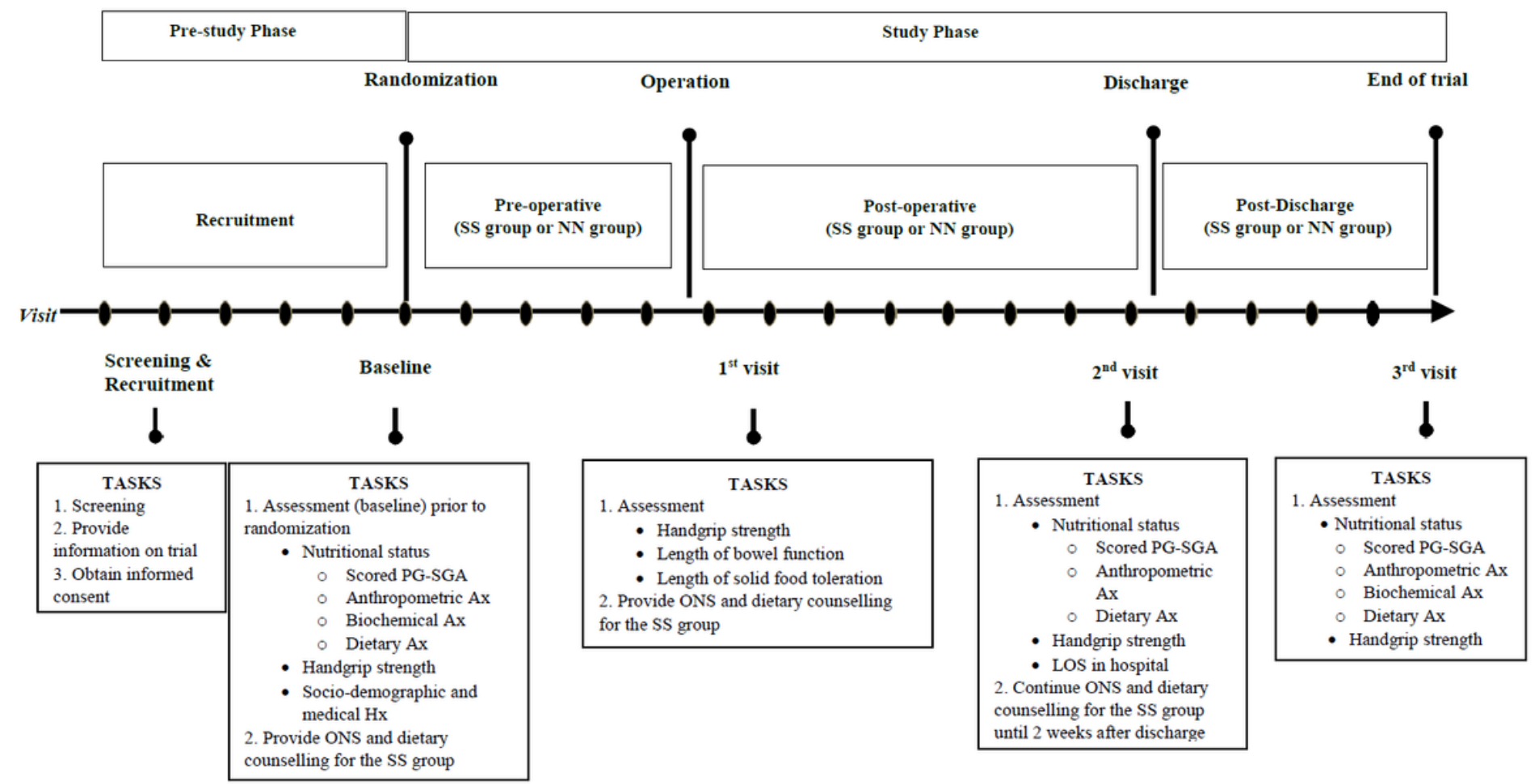

Figure 2

A graphical presentation of the protocol and timeline of the intervention study Ax: Assessment; $\mathrm{Hx}$ : History; PG-SGA: Patient-Generated Subjective Global Assessment; ONS: Oral Nutrition Support; LOS: Length of Stay; SS: Intervention Group; NN: Control Group

\section{Supplementary Files}

This is a list of supplementary files associated with this preprint. Click to download.

- SPIRITCHECKLIST.docX 